\title{
Quantum Dots: Need to Explore Potential for Delivery of Drugs and Biomolecules
}

\section{Galgatte UC*}

Department of Pharmaceutics, Modern College of Pharmacy, India

*Corresponding author: Upendra C Galgatte, Associate Professor, Department of Pharmaceutics, Modern College of Pharmacy, Nigdi, Pune- 411044, India, Tel: +919890755938; E-mail: ucgpharm@rediffmail.com

\section{Editorial}

Volume 1 Issue 8

Received Date: November 30, 2017

Published Date: December 15, 2017

\section{Editorial}

Quantum dots (QDs) are the semiconductor nanoparticles having the range of $2 \mathrm{~nm}$ to $10 \mathrm{~nm}$ [1]. One of the properties of semiconductor metals of the group IV to group VI of periodic table, to show fluorescence is established in the biomedical research for quantitative fluorescence imaging and detection [2]. QDs based bioimaging is widely used. Comparing with other nanoparticles, a distinct property of QDs is excitation of multiple color fluorescence based on particle size. QDs emit the same wavelength of light which is used as excitation wavelength. Therefore with single excitation light source, multiple QDs with different spectra can be observed. The term quantum dot was coined by Mark Reed.

The physics of a semiconductor metal is found to be helpful in the biomedical research. However, likewise, bio imaging properties, it is the need to explore the potential of quantum dots to deliver active pharmaceutical ingredients at the site. QDs can be explored for their potential to target the delivery as well as possibility to track the delivery inside the body. This can be utilized to understand bio-distribution of pharmaceutical active ingredients after administration into the body. In addition to its earlier role, it would be possible to target the drugs and to estimate them quantitatively at the site of action. Thus dual role of quantum dots would be appreciable.

\section{Synthesis of QDs}

Commonly used methods for preparation of QDs are colloidal synthesis, lithographic technique, fabrication, viral assembly, electrochemical assembly etc [3]. Besides, the methods reported for synthesis of QDs, green synthesis is also possible. Mariselvam R, et al. [4] reported green synthesis of copper QDs by using root extracts of plant Rubia cardifolia having a particle size $22.68 \mathrm{~nm}$. The copper QDs were tested for antibacterial properties. Vidhya K, et al. [5] reported green synthesis of glucose capped $\mathrm{ZnO}: \mathrm{Fe}$ QDs and used to test antibacterial properties. Vidhya $K$, et al. [6] similarly, also reported green synthesis of manganese doped, sucrose capped $\mathrm{ZnO}$ multifunctional QDs for anticancer activity having the particle size of $12 \mathrm{~nm}$.

\section{Structural Properties and Delivery Potential}

Few years back, some of the researchers have carried out the synthesis of quantum dots from its source metal and characterized their particulate properties. The most important property found so far the changing color fluorescence with the particle size. It has been found that the QDs show blue color for the size range of up to 5-10 $\mathrm{nm}$. This size generally increases to $20 \mathrm{~nm}$ after polymer application.

The quantum dots below the size of $5 \mathrm{~nm}$ are usually filtered and cleared by kidneys. However, relatively larger particles reach to the target site after passing through reticuloendothelial system. Hence the role of optimum particle size is important to evaluate and trace out delivery potential. Other supportive physical properties of quantum dots include narrow size distribution, good flowability, more resistant to degradation than other optical imaging probes. Quantum dots have greater photo stability due to their inorganic nature than other dyes [3] and long fluorescent lifetime after excitation [7]. The 


\section{Open Access Journal of Pharmaceutical Research}

inorganic core; consist of either of semiconductor metal mentioned above serves as imaging contrast agent, above which hydrophobic drug molecules are encapsulated by amphiphilic polymer. The hydrophilic drugs are immobilized on the other side of amphiphilic polymer which is hydrophilic in nature. The hydrophilic drugs and biomolecules are bounded by covalent or non-covalent bonds. This is depicted in Figure 1. Water soluble quantum dots can be designed with amphiphilic polymer, amphiphilic polymer conjugated with poly (ethylene glycol) or encapsulation with block co-polymer micelles [8]. Quantum dots are administered as a colloidal solution through intravenous route, they reach to target and due to their fluorescence, they can be detected.

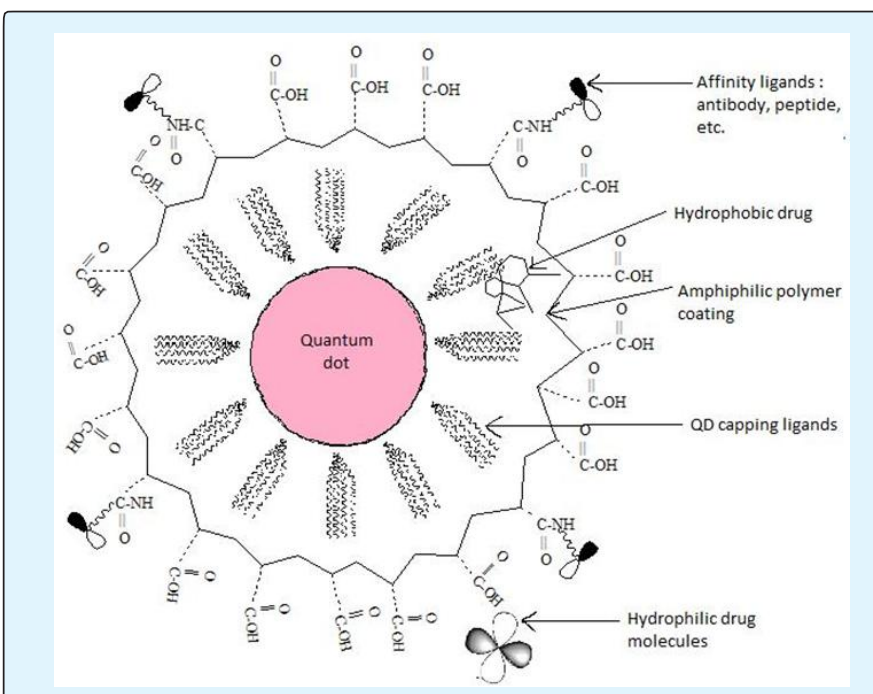

Figure 1: Typical structure of QD showing attachment of ligands, hydrophilic and hydrophobic molecules.

Ketoprofen and Dexketoprofen coated Zno quantum dots of size smaller than $10 \mathrm{~nm}$ were prepared for transdermal delivery. The permeability coefficients were estimated by using rat skin. It was observed that the permeability coefficient of Ketoprofen coated Zno quantum dots and Dexketoprofen coated Zno quantum dots were considerably higher than their aqueous solutions respectively [9]. Chakravarthy KV, et al. [10] have reported the ability of nanoconjugates of CdSe/CdS/ZnS quantum dots and doxorubicin to target alveolar macrophages. In the confocal study it was observed that alveolar macrophages cell nucleus and induction of apoptosis. Doxorubicin was showing its activity after coupling with nanoparticles. Ultimately the results demonstrated targeted macrophage selective therapy for the treatment of pulmonary disease. Godbole $\mathrm{N}$, et al. [11] reported quantum dots as carrier for delivery of 5-flurouracil. Eudragit EPO coated $\mathrm{ZnO}$ quantum dots having mean particle size of $201.92 \mathrm{~nm}$ and zeta potential of $+1.85 \mathrm{mv}$ could be synthesized successfully by colloidal synthesis method.

Now the scientists are concentrating on the delivery of functional genes for therapeutic use by quantum dots. Gene delivery systems are mainly viral and nonviral based. However, non-viral is efficient and safe vector for gene delivery. Surface modifications of QDs hold promising approach for traceable gene delivery. For intracellular delivery and real-time imaging of siRNA into cancer cells with significantly reduced cytotoxicity has been reported with Cd-Se QD-amphipol technology, QDpeptide conjugates and 2-vinyl pyridine functionalized silicon quantum dots were designed for SiRNA transfection and therapeutic imaging $[12,13]$. Cysteamine capped, water soluble CdTe QD vectors, conjugated with plasmid DNA through electrostatic interaction, have been reported. QD-DNA complexes are capable for controllable release of DNA and gene expression in HEK293 cells in the visible mode [14].

Chitosan encapsulated $\mathrm{ZnO}$ quantum dots are smart nanocarriers for drug delivery. Chitosan is a natural polymer with $\mathrm{N}$-acetyl glucosamine and D-glucosamine, with one amino group and two hydroxyl groups, is promising as to encapsulate quantum dots. Chitosan enables many properties suitable for encapsulation and delivery system. To note, water solubility, chelation with metal ions, easy ligand attachment, biocompatibility, ease of processing, strong electrostatic interaction with negatively charged biomolecules and quantum dots are few of them [15]. ZnO-chitosan-folate system has been used as a nanocarrier for delivery of doxorubicin, an antineoplastic agent used in tumor treatments, through physical and chemical interactions. The release of doxorubicin from $\mathrm{ZnO}-\mathrm{QD}$-chitosan-folate carrier is due to presence of folic acid that weakens the electrostatic interaction between doxorubicin and ZnO QDs [16]. Muhammad F, et al. [17] have evaluated ZnO QDs as a platform for targeted and $\mathrm{pH}$ responsive intracellular delivery of doxorubicin. Doxorubicin release to the cytosol is favored due to the weakened interaction between $\mathrm{Zn}^{2+}$ and doxorubicin in acidic conditions of dissolution. ZnO QDs have shown strong activity against some of Gram- positive and Gram-negative bacteria and biocompatibility with colloidal semiconductor luminescent inorganic materials [18].

Comparing the properties of other nanoparticles used for drug delivery, QDs possess additional property to target the receptors and to help to establish the concentration at the site by bio-imaging. 


\section{Open Access Journal of Pharmaceutical Research}

\section{Water soluble QDs}

To obtain biologically active and compatible QDs, newly synthesized QDs are functionalized by applying secondary coat that ensures core durability, water solubility. Water soluble QDs are compact and usually coated with monolayer of any hydrophilic surfactant. This hydrophilic surfactant prevents aggregation and promotes dispersion in aqueous medium. Different surfactants with different terminal groups allow different conjugation of biomolecules. The choice of end group includes primary amines, carboxylic acid and diols. Libin Tang, et al. [19] reported glucose derived water soluble crystalline quantum dots with average diameter of 1.65 $\mathrm{nm}$ by microwave assisted method. Yan $\mathrm{Li}$, et al. [20] reported QDs stable at pH 2-13, water soluble capped by poly (ethylene glycol) modified dithiocarbamate. The poly ethylene segment makes QDs water soluble. Apart from the delivery aspect of water stable quantum dots, its analytical use is admirable. Casa $M$, et al. [21] have synthesized water stable $\mathrm{ZnO}$ quantum dots by simple method by using green solvent at low temperature for detection of aniline compounds in water.

\section{Oil soluble QDs}

Oil soluble quantum dots have been prepared by Zeng $\mathrm{R}$, et al. [22] $\mathrm{Au}: \mathrm{ZnCdS}$ and $\mathrm{Au}: \mathrm{Zn} \mathrm{CdS/ZnS} \mathrm{core/shell}$ QDs were prepared with tunable emission color. $\mathrm{Au}^{+}$is used as primary dopant and trivalent cation is used as $\operatorname{In}^{3+}$ as co-dopant. This strategy may be useful to control optical properties.

\section{Future Perspectives}

QDs may produce immunogenic reactions [23]. They may be ineffective as a result of antibody binding. On the other hand, the metal such as cadmium produce liver toxicity due to it's unlike renal filtration. Cadmium telluride (Cd-Te) QDs are toxic. They are used as fluorescent probes for biological imaging and to track drug targeting. However, researchers were using gelatin in the process of its preparation to minimize toxicity [24]. Therefore, selection of source metal should be optimum. In this regard, Zno quantum dots are gaining much more attention. Thus, minimizing the toxicity would be one of the marked targets in future. None the less, the large numbers of clinical trials are needed to be carried out to improve efficiency and safety of quantum dots. One more critical factor which we must consider that determines the cytotoxicity of QDs is possibility of the leakage of a metal from the core due to photolysis or oxidation [25]. Like drug delivery, use of QDs in drug and chemical analytical field looks emerging in future.

\section{References}

1. Xing Y, Rao J (2008) Quantum dot bioconjugates for in vitro diagnostics \& in vivo imaging. Cancer Biomark 4(6): 307-319.

2. Azzazy HM, Mansour MM, Kazmierczak SC (2007) From diagnostics to therapy: Prospects of quantum dots. Clin Biochem 40(13-14): 917-927.

3. Modani S, Kharwade M, Nijhawan M (2013) Quantum dots: A novelty of medical field with multiple applications. Int J Curr Pharm Res 5(4): 55-59.

4. Mariselvam R, Ranjitsingh AJA, Padmalatha C, Mosae Selvakumar P (2014) Green synthesis of copper QDs using Rubia cradifolia plant root extracts and its antibacterial properties. JAIR 3(4): 191-194.

5. Vidya K, Bhoopathi G, Devarajn V P, Saravanan M (2014) Green synthesis of glucose capped Zno:Fe quantum dots: A study on structural, optical properties and application. Res J Recent Sci 3: 238241.

6. Vidhya K, Saravananb M, Devarajanc VP, Satheeskumard S, Ashraf Alie M, et al. (2014) Green synthesis and anti-cancer activity of multifunctional ZnO:Mn-natural biomolecule quantum dots system. Malaya Journal of Biosciences 1(4): 267-272.

7. Timothy J, Bakhshi R, Petrova D, Pocock R, Imani M, et al. (2007) Biological applications of quantum dots. Biomaterials 28: 4717-4732.

8. Ghasemi Y, Peymani P, Afifi S (2009) Quantum dot: magic nanoparticle for imaging, detection and targeting. Acta Biomed 80(2): 156-165.

9. Degim IT, Kodiglu D (2013) Cheap, suitable predictable and manageable nanoparticles for drug delivery: Quantum dots. Curr Drug Deliv 10(1): 3238.

10. Chakravarthy KV, Davidson BA, Helinski JD, Ding H, Law WC, et al. (2011) Doxorubincin-conjugated quantum dots to target alveolar macrophages and inflammation. Nanomedicine 7(1): 88-96.

11. Godbole NN, Galgatte UC, Chaudhari PD (2016) Development and in vitro evaluation of Quantum dots as carrier for delivery of 5-flurouracil. Int J Pharm Pharm Sci 8(1): 289-296. 


\section{Open Access Journal of Pharmaceutical Research}

12. Qi L, Gao X (2008) Quantum dot-Amphipol nanocomplex for intracellular delivery and real time imaging of siRNA. ACS Nano 2(7): 1403-1410.

13. Walther C, Meyer K, Rennert R, Neundorf I (2008) Quantum dot-carrier peptide conjugates suitable for imaging and delivery applications. Bioconjug Chem 19(12): 2346-2356.

14. Li D, Li G, Guo W, Li P, Wang E, et al. (2008) Glutathione-mediated release of functional plasmid DNA from positively charged quantum dots. Biomaterials 29(18): 2776-2782.

15. Gulia S, Kakkar R (2013) Zno quantum dots for biomedical applications. Adv Mat Lett 4(12): 876-887.

16. Yuan Q, Hein S, Misra RD (2010) New generation of chitosan-encapsulated $\mathrm{ZnO}$ quantum dots loaded with drug: synthesis, characterization, and in vitro drug delivery response. Acta Biomater 6(7): 2732-2739.

17. Muhammad F, Mingyi Guo, Yingjie Guo, Wenxiu Qi, Fengyu $\mathrm{Qu}$, et al. (2011) Acid degradable $\mathrm{ZnO}$ quantum dots as a platform for targeted delivery of an anticancer drug. J Mater Chem 21: 13406-13412.

18. Zahra F, Harsini MF, Chalabian F, Katouzian F, Shafiekhani A, et al. (2013) Influence of Modified ZnO Quantum Dots and Nanostructures as New Antibacterials. ANP 2(3): 247-258.
19. Tang L, Ji R, Cao X, Lin J, Jiang H, et al. (2012) Deep ultraviolet photoluminescence of water soluble selfpassivated graphene quantum dots. ACS Nano 6(6): 5102-5110.

20. Li Y, Shen B, Liu L, Xu H, Zhong X (2012) Stable water soluble quantum dots capped by poly (ethylene glycol) modified dithiocarbamate. Colloids Surf A Physicochem Eng Asp 410: 144-152.

21. Casa M, Sarno M, Paciello L, Beaumont MR, Ciambelli $P$ (2016) Synthesis and characterization of water stable Zno quantum dot based sensor for nitro organic compounds. Chemical Engineering Transactions 47: 7-12.

22. Zeng R, Sun Z, Zhou C, Fang C, Han GC, et al. (2016) Well resolved oil soluble Au-dopped ZnCdS quantum dots and enhancing doping emission with Incodoping. J Alloys Compd 671: 66-73.

23. Lijia S, Yanfang G, Feng Y (2011) Semiconductor Quantum Dots for Biomedicial Applications. Sensors 11(12): 11736-11751.

24. Dey NS, Bhanoji Rao ME (2011) Quantum dot: novel carrier for drug delivery. International Journal of Research in Pharmaceutical and Biomedical Sciences 2(2): 448-458.

25. Shan J, Yanxi Hu, Zhangun Gu, Lei Liu, Hai Chen-Wu (2011) Application of quantum dots in biological imaging. J Nanomater 834139: 13. 